\title{
A maximum principle for stochastic differential games with $g$-expectations and partial information
}

\author{
Ta Thi Kieu $\mathrm{An}^{1}$ and Bernt Øksendal ${ }^{1,2}$
}

3 November 2010

\begin{abstract}
In this paper, we initiate a study on optimal control problem for stochastic differential games under generalized expectation via backward stochastic differential equations and partial information. We first prove a sufficient maximum principle for zero-sum stochastic differential game problems. And then extend our approach to general stochastic differential games (nonzero-sum games), and obtain an equilibrium point of such game. Finally we give some examples of applications.
\end{abstract}

Key words: Jump diffusion, stochastic control, stochastic differential game, forward-backward stochastic differential equations, $g$-expectation, sufficient maximum principle.

MSC2010: 93E20, 91A15, 91A23, 91B30, 91G80, 60G51, 60H07, 60H20, 60H30, $60 \mathrm{~J} 75$

\section{Introduction}

Suppose the dynamics of a stochastic system is described by a stochastic differential equation on a complete filtered probability space $\left(\Omega, \mathcal{F},\left\{\mathcal{F}_{t}\right\}_{t \geq 0}, \mathbb{P}\right)$ of

\footnotetext{
${ }^{1}$ Centre of Mathematics for Applications (CMA), Department of Mathematics, University of Oslo, P. O. Box 1053, Blindern, N-0316 Oslo, Norway.

The research leading to these results has received funding from the European Research Council under the European Community's Seventh Framework Programme (FP7/2007-2013)/ERC grant agreement no [228087].

Email: <atkieu@math.uio.no>,<oksendal@math.uio.no>

2 Norwegian School of Economics and Business Administration, Helleveien 30, N-5045 Bergen, Norway.
} 
the form:

$$
\begin{aligned}
d X(t) & =b\left(t, X(t), u_{0}(t)\right) d t+\sigma\left(t, X(t), u_{0}(t)\right) d W(t) \\
& +\int_{\mathbb{R}_{0}} \gamma\left(t, X\left(t^{-}\right), u_{1}\left(t^{-}, z\right), z\right) \widetilde{N}(d t, d z), t \in[0, T], \\
X(0) & =x \in \mathbb{R}^{n} .
\end{aligned}
$$

Here $b:[0, T] \times \mathbb{R}^{n} \times K \rightarrow \mathbb{R}^{n} ; \sigma:[0, T] \times \mathbb{R}^{n} \times K \rightarrow \mathbb{R}^{n \times n}$ and $\gamma:$ $[0, T] \times \mathbb{R}^{n} \times K \times \mathbb{R}_{0} \rightarrow \mathbb{R}^{n \times n}$ are given continuous functions, and $W(t)$ is $n$-dimensional Brownian motion, $\widetilde{N}(\cdot, \cdot)$ are $n$ independent compensated Poisson random measures and $K$ is a given closed subset of $\mathbb{R}^{n}$. The processes $u_{0}(t)=u_{0}(t, \omega)$ and $u_{1}(t)=u_{1}(t, z, \omega), \omega \in \Omega$ are our control processes. We assume that $u_{0}(t), u_{1}(t, z)$ have values in a given set $K$ for a.a. $t, z$ and adapted to a given filtration $\left\{\mathcal{G}_{t}\right\}_{t \in[0, T]}$, where

$$
\mathcal{G}_{t} \subseteq \mathcal{F}_{t} ; \quad t \in[0, T] .
$$

For example, we could have a delayed information flow of the form

$$
\mathcal{G}_{t}=\mathcal{F}_{(t-\delta)^{+}} ; \quad t \in[0, T],
$$

where $(t-\delta)^{+}=\max (0, t-\delta)$ and $\delta>0$ is a given constant. We call $u=\left(u_{0}, u_{1}\right)$ an admissible control if (1) has a unique strong solution.

Let $f:[0, T] \times \mathbb{R}^{n} \times K \rightarrow \mathbb{R}$ be a continuous function, namely the profit rate, and $h: \mathbb{R}^{n} \rightarrow \mathbb{R}$ be a concave function, namely the bequest function. If $u$ is an admissible control we define the performance criterion $J(u)$ by

$$
J(u)=\mathbb{E}\left[\int_{0}^{T} f(t, X(t), u(t)) d t+h(X(T))\right] .
$$

Now suppose that the controls $u_{0}(t)$ and $u_{1}(t, z)$ have the form

$$
\begin{aligned}
u_{0}(t) & =\left(\theta_{0}(t), \pi_{0}(t)\right) ; \quad t \geq 0, \\
u_{1}(t, z) & =\left(\theta_{1}(t, z), \pi_{1}(t, z)\right) ; \quad(t, z) \in[0, \infty) \times \mathbb{R}^{n} .
\end{aligned}
$$

We let $\Theta_{\mathcal{G}}$ and $\Pi_{\mathcal{G}}$ be given families of admissible controls $\theta=\left(\theta_{0}, \theta_{1}\right)$ and $\pi=\left(\pi_{0}, \pi_{1}\right)$, respectively.

Problem 1. The classical partial information zero-sum stochastic differential game problem is to find $\left(\theta^{*}, \pi^{*}\right) \in \Theta_{\mathcal{G}} \times \Pi_{\mathcal{G}}$ such that

$$
J\left(\theta^{*}, \pi^{*}\right)=\sup _{\pi \in \Pi_{\mathcal{G}}}\left(\inf _{\theta \in \Theta_{\mathcal{G}}} J(\theta, \pi)\right) .
$$

Such a control $\left(\theta^{*}, \pi^{*}\right)$ is called an optimal control (if it exists).

The intuitive idea is that there are two players, $I$ and $I I$. Player $I$ controls $\theta:=\left(\theta_{0}, \theta_{1}\right)$ and player $I I$ controls $\pi:=\left(\pi_{0}, \pi_{1}\right)$. The actions of the players 
are antagonistic, which means that between $I$ and $I I$ there is a payoff $J(\theta, \pi)$ which is a cost for $I$ and a reward for $I I$.

Problem 1 for jumps was studied recently by several authors, e.g. [1], [2], [6], [8] and references therein. In this paper we study this game in the case when the performance criterion $J(u)$ in $(2)$ is replaced by a criterion involving risk. If we interpret risk in the sense of a convex risk measure, it can be represented as a nonlinear expectation called $g$-expectation. See [4], [5], [9], [10] and [11] for more information about this. More precisely, let

$$
g:[0, T] \times \mathbb{R}^{n} \times \mathbb{R}^{n} \times L^{2}(\nu) \rightarrow \mathbb{R}
$$

be a given convex function such that $g$ is uniformly Lipschitz with respect to $(y, k, l)$, i.e.,

$$
\left|g(t, y, k, l)-g\left(t, y^{\prime}, k^{\prime}, l^{\prime}\right)\right| \leq K\left(\left|y-y^{\prime}\right|+\left|k-k^{\prime}\right|+\left|l-l^{\prime}\right|\right),
$$

and such that, for each $T>0,(y, k, l) \in\left(\mathbb{R}^{n} \times \mathbb{R}^{n} \times L^{2}(\nu)\right), g(t, y, k, l)$ is progressively measurable.

Let $\mathbb{F}$ be a family of $\mathcal{F}_{T^{-}}$measurable random variables $\xi: \Omega \rightarrow \mathbb{R}^{n}, \xi \in \mathbb{L}^{2}(\mathbb{P})$ where $T>0$ is a fixed constant. Consider the following backward stochastic differential equation (BSDE, for short):

$$
\begin{aligned}
d Y(t) & =-g(t, K(t), L(t, \cdot)) d t+K(t) d W(t)+\int_{\mathbb{R}_{0}} L(t, z) \tilde{N}(d t, d z), \\
Y(T) & =\xi .
\end{aligned}
$$

We then define

Definition 1.1. For each $\xi \in \mathbb{F}$, we call

$$
\mathcal{E}_{g}(\xi):=Y(0)
$$

the $g$-expectation of $\xi$ related to $g$.

One can show that the map $\xi \rightarrow \mathcal{E}_{g}(\xi)$ keeps all the properties that $\mathbb{E}$ has, except possibly the linearity. Further, it is clear that when $g(\cdot)=0, \mathcal{E}_{g}$ is reduced to the classical expectation $\mathbb{E}$.

With the above defined generalized expectation, we now introduce the following performance functional

$$
J_{g}(\theta, \pi)=\mathcal{E}_{g}\left[\int_{0}^{T} f(t, X(t), u(t)) d t+h(X(T))\right] .
$$

We can formulate our problem with generalized expectation as follows

Problem 2. Find $\left(\theta^{*}, \pi^{*}\right) \in \Theta_{\mathcal{G}} \times \Pi_{\mathcal{G}}$ such that

$$
J_{g}\left(\theta^{*}, \pi^{*}\right)=\sup _{\pi \in \Pi_{\mathcal{G}}}\left(\inf _{\theta \in \Theta_{\mathcal{G}}} J_{g}(\theta, \pi)\right) .
$$


This problem can be expressed in a different way. Let $(\eta(\cdot), \zeta(\cdot), \beta(\cdot, \cdot))$ be adapted solution of following BSDE:

$$
\left\{\begin{array}{l}
d \eta(t)=-g(t, \zeta(t), \beta(t, \cdot)) d t+\zeta(t) d W(t)+\int_{\mathbb{R}_{0}} \beta(t, z) \widetilde{N}(d t, d z) \\
\eta(T)=\xi(x, \theta, \pi)
\end{array}\right.
$$

where

$$
\xi(x, \theta, \pi)=\int_{0}^{T} f(t, X(t), u(t)) d t+h(X(T)) .
$$

Define

$$
\left\{\begin{array}{l}
Y(t)=\eta(t)-\int_{0}^{t} f(t, X(t), u(t)) d t \\
K(t)=\zeta(t), \quad L(t, z)=\beta(t, z) .
\end{array}\right.
$$

Then $(Y(\cdot), K(\cdot), L(\cdot))$ is the unique adapted solution of the following BSDE:

$$
\left\{\begin{aligned}
d Y(t)= & -[g(t, K(t), L(t, \cdot))+f(t, X(t), u(t))] d t \\
& +K(t) d W(t)+\int_{\mathbb{R}_{0}} L(t, z) \widetilde{N}(d t, d z) \\
Y(T)= & h(X(T))
\end{aligned}\right.
$$

Thus, our problem can be formulated as follows: The state process $(X(t), Y(t))$ of our system is described by the following coupled forward-backward stochastic differential equation (FBSDE):

$$
\left\{\begin{aligned}
d X(t)= & b\left(t, X(t), u_{0}(t)\right) d t+\sigma\left(t, X(t), u_{0}(t)\right) d W(t) \\
& +\int_{\mathbb{R}_{0}} \gamma\left(t, X\left(t^{-}\right), u_{1}\left(t^{-}, z\right), z\right) \widetilde{N}(d t, d z), \\
d Y(t)= & -[g(t, K(t), L(t, \cdot))+f(t, X(t), u(t))] d t \\
& +K(t) d W(t)+\int_{\mathbb{R}_{0}} L(t, z) \widetilde{N}(d t, d z), \\
X(0)= & X_{0}, \quad Y(T)=h(X(T)),
\end{aligned}\right.
$$

and the cost function is given of the form:

$$
\begin{aligned}
& J_{g}(\theta, \pi)=Y(0) \\
& =\mathbb{E}\left[h(X(T))+\int_{0}^{T}(g(t, K(t), L(t, \cdot))+f(t, X(t), u(t))) d t\right] .
\end{aligned}
$$

The problem is to find $u^{*}=\left(\theta^{*}, \pi^{*}\right) \in \Theta_{\mathcal{G}} \times \Pi_{\mathcal{G}}$ such that

$$
J_{g}\left(\theta^{*}, \pi^{*}\right)=\sup _{\pi \in \Pi_{\mathcal{G}}}\left(\inf _{\theta \in \Theta_{\mathcal{G}}} J_{g}(\theta, \pi)\right) .
$$

The paper is organized as follows: In Section 2 we study the partial optimal control problem for zero-sum stochastic differential games with $g$-expectations and we prove a partial information sufficient maximum principle for such a problem. In Section 3 we generalize our approach to the general case, not necessarily of zero-sum type, and also give an equilibrium point for nonzerosum games. Finally, in Section 4 we apply our results to finance market. 


\section{A maximum principle for zero-sum games with $g$-expectations}

We now present a maximum principle for problem (13).

The Hamiltonian

$$
H:[0, T] \times \mathbb{R}^{n} \times \mathbb{R}^{n} \times \mathbb{R}^{n} \times L^{2}(\nu) \times K_{1} \times K_{2} \times \mathbb{R}^{n} \times \mathbb{R}^{n} \times \mathbb{R}^{n} \times L^{2}(\nu)
$$

is defined by

$$
\begin{aligned}
& H(t, x, y, k, l, \theta, \pi, \mu, \varphi, \psi, \phi)=g(t, k, l)+f(t, x, \theta, \pi) \\
& +(g(t, k, l)+f(t, x, \theta, \pi)) \mu+b(t, x, \theta, \pi) \varphi \\
& +\sigma(t, x, \theta, \pi) \psi+\int_{\mathbb{R}_{0}} \gamma(t, x, \theta, \pi, z) \phi(t, z) \nu(d z) .
\end{aligned}
$$

We assume that $H$ is differentiable in the variables $x, y, k, l$. The adjoint equation in the unknown adapted processes $\mu, \varphi, \psi, \phi$ is the following FBSDE:

$$
\left\{\begin{aligned}
d \mu(t)= & \frac{\partial H}{\partial y}(t, X(t), Y(t), K(t), L(t), \theta(t), \pi(t), \mu(t), \varphi(t), \psi(t), \phi(, \cdot)) d t \\
+ & \frac{\partial H}{\partial k}(t, X(t), Y(t), K(t), L(t), \theta(t), \pi(t), \mu(t), \varphi(t), \psi(t), \phi(, \cdot)) d W(t) \\
+ & \int_{\mathbb{R}_{0}} \nabla_{l} H(t, X(t), Y(t), K(t), L(t), \theta(t), \pi(t), \mu(t), \varphi(t), \psi(t), \phi(, \cdot)) \widetilde{N}(d t, d z), \\
& = \\
& +\psi(t) d W(t)+\int_{\mathbb{R}_{0}} \phi(t, z) \widetilde{N}(d t, d z), \\
d \varphi(t)= & 0, \quad \varphi(T)=(1+\mu(T)) h^{\prime}(X(T)),
\end{aligned}\right.
$$

where $\nabla_{l} H$ denotes the gradient (Frechet derivative) of $H$ with respect to $l$.

With a slight abuse of notation we will let $\Theta$ and $\Pi$ denote given sets of possible control values of $\theta(t)$ and $\pi(t), t \in[0, T]$, respectively.

Theorem 2.1. Let $(\hat{\theta}, \hat{\pi}) \in \Theta_{\mathcal{G}} \times \Pi_{\mathcal{G}}$ with corresponding solutions $\hat{X}(t), \hat{Y}(t)$, $\hat{K}(t), \hat{L}(t, z), \hat{\mu}(t), \hat{\varphi}(t), \hat{\psi}(t), \hat{\phi}(t, z)$ of equations (11) and (15). Suppose that

(The conditional minimum principle)

$$
\begin{aligned}
& \inf _{\theta \in \Theta} \mathbb{E}\left[H(t, \hat{X}(t), \hat{Y}(t), \hat{K}(t), \hat{L}(t, \cdot), \theta, \hat{\pi}(t), \hat{\mu}(t), \hat{\varphi}(t), \hat{\psi}(t), \hat{\phi}(t, \cdot)) \mid \mathcal{G}_{t}\right] \\
& =\mathbb{E}\left[H(t, \hat{X}(t), \hat{Y}(t), \hat{K}(t), \hat{L}(t, \cdot), \hat{\theta}(t), \hat{\pi}(t), \hat{\mu}(t), \hat{\varphi}(t), \hat{\psi}(t), \hat{\phi}(t, \cdot)) \mid \mathcal{G}_{t}\right] \\
& =\sup _{\pi \in \Pi} \mathbb{E}\left[H(t, \hat{X}(t), \hat{Y}(t), \hat{K}(t), \hat{L}(t, \cdot), \hat{\theta}(t), \pi, \hat{\mu}(t), \hat{\varphi}(t), \hat{\psi}(t), \hat{\phi}(t, \cdot)) \mid \mathcal{G}_{t}\right] .
\end{aligned}
$$

(i) Suppose that, for all $t \in[0, T], h(x)$ is concave and

$$
(x, y, k, l, \pi) \rightarrow H(t, x, y, k, l, \hat{\theta}(t), \pi, \hat{\mu}(t), \hat{\varphi}(t), \hat{\psi}(t), \hat{\phi}(t, \cdot))
$$

is concave. Then

$$
J_{g}(\hat{\theta}, \hat{\pi}) \geq J_{g}(\hat{\theta}, \pi), \quad \text { for all } \pi \in \Pi_{\mathcal{G}}
$$


and

$$
J_{g}(\hat{\theta}, \hat{\pi})=\sup _{\pi \in \Pi_{\mathcal{G}}} J_{g}(\hat{\theta}, \pi) .
$$

(ii) Suppose that, for all $t \in[0, T], h(x)$ is convex and

$$
(x, y, k, l, \theta) \rightarrow H(t, x, y, k, l, \theta, \hat{\pi}(t), \hat{\mu}(t), \hat{\varphi}(t), \hat{\psi}(t), \hat{\phi}(t, \cdot))
$$

is convex. Then

$$
J_{g}(\hat{\theta}, \hat{\pi}) \leq J_{g}(\theta, \hat{\pi}), \quad \text { for all } \theta \in \Theta_{\mathcal{G}}
$$

and

$$
J_{g}(\hat{\theta}, \hat{\pi})=\inf _{\theta \in \Theta_{\mathcal{G}}} J_{g}(\theta, \hat{\pi}) .
$$

(iii) If both cases (i) and (ii) hold (which implies, in particular, that $h$ is an affine function), then $\left(\theta^{*}, \pi^{*}\right):=(\hat{\theta}, \hat{\pi})$ is an optimal control and

$$
J_{g}(\hat{\theta}, \hat{\pi})=\sup _{\pi \in \Pi_{\mathcal{G}}}\left(\inf _{\theta \in \Theta_{\mathcal{G}}} J_{g}(\theta, \pi)\right)=\inf _{\theta \in \Theta_{\mathcal{G}}}\left(\sup _{\pi \in \Pi_{\mathcal{G}}} J_{g}(\theta, \pi)\right) .
$$

Proof. i) Choose $(\theta, \pi) \in \Theta_{\mathcal{G}} \times \Pi_{\mathcal{G}}$ with corresponding solutions $X(t), Y(t)$, $K(t), L(t, z), \mu(t), \varphi(t), \psi(t)$ and $\phi(t, z)$. In the following we write

$$
\begin{aligned}
\hat{H}(t) & =H(t, \hat{X}(t), \hat{Y}(t), \hat{K}(t), \hat{L}(t, \cdot), \hat{\theta}(t), \hat{\pi}(t), \hat{\mu}(t), \hat{\varphi}(t), \hat{\psi}(t), \hat{\phi}(t, \cdot)), \\
H^{\hat{\theta}}(t) & =H\left(t, X^{\hat{\theta}}(t), Y^{\hat{\theta}}(t), K^{\hat{\theta}}(t), L^{\hat{\theta}}(t), \hat{\theta}(t), \pi(t), \hat{\mu}(t), \hat{\varphi}(t), \hat{\psi}(t), \hat{\phi}(t, \cdot)\right), \\
H^{\hat{\pi}}(t) & =H\left(t, X^{\hat{\pi}}(t), Y^{\hat{\pi}}(t), K^{\hat{\pi}}(t), L^{\hat{\pi}}(t), \theta(t), \hat{\pi}(t), \hat{\mu}(t), \hat{\varphi}(t), \hat{\psi}(t), \hat{\phi}(t, \cdot)\right)
\end{aligned}
$$

and similarly with $\hat{f}(t), f^{\hat{\theta}}(t), f^{\hat{\pi}}(t) \ldots$ etc. Then

$$
J_{g}(\hat{\theta}, \hat{\pi})-J_{g}(\hat{\theta}, \pi)=I_{1}+I_{2},
$$

where

$$
I_{1}=\mathbb{E}\left[\int_{0}^{T}\left(\hat{g}(t)-g^{\hat{\theta}}(t)+\hat{f}(t)-f^{\hat{\theta}}(t)\right) d t\right]
$$

and

$$
I_{2}=\mathbb{E}\left[h(\hat{X}(T))-h\left(X^{\hat{\theta}}(T)\right)\right] .
$$

By the definition of $H$ we have

$$
\begin{aligned}
I_{1}=\mathbb{E}\left[\int_{0}^{T}\right. & \left\{\hat{H}(t)-H^{\hat{\theta}}(t)-\left(\hat{g}(t)-g^{\hat{\theta}}(t)+\hat{f}(t)-f^{\hat{\theta}}(t)\right) \mu(t)\right. \\
& -\left(\hat{b}(t)-b^{\hat{\theta}}(t)\right) \hat{\varphi}(t)-\left(\hat{\sigma}(t)-\sigma^{\hat{\theta}}(t)\right) \hat{\psi}(t) \\
& \left.\left.-\int_{\mathbb{R}_{0}}\left(\hat{\gamma}(t)-\gamma^{\hat{\theta}}(t)\right) \hat{\phi}(t, z) \nu(d z)\right\} d t\right] .
\end{aligned}
$$


Since $\hat{\mu}(0)=0$, we can rewrite $I_{2}$ as following:

$$
I_{2}=\mathbb{E}\left[h(\hat{X}(T))-h\left(X^{\hat{\theta}}(T)\right)+\left(\hat{Y}(0)-Y^{\hat{\theta}}(0)\right) \hat{\mu}(0)\right] .
$$

By the Itô formula, we have

$$
\begin{aligned}
& \mathbb{E}\left[\left(\hat{Y}(0)-Y^{\hat{\theta}}(0)\right) \hat{\mu}(0)\right]=\mathbb{E}\left[\left(\hat{Y}(T)-Y^{\hat{\theta}}(T)\right) \hat{\mu}(T)\right] \\
& \quad-\mathbb{E}\left[\int _ { 0 } ^ { T } \left\{\left(\hat{Y}(t)-Y^{\hat{\theta}}(t)\right) d \hat{\mu}(t)+\hat{\mu}(t) d\left(\hat{Y}(t)-Y^{\hat{\theta}}(t)\right)\right.\right. \\
& \left.\left.\quad+\frac{\partial \hat{H}}{\partial k}(t)\left(\hat{K}(t)-K^{\hat{\theta}}(t)\right)+\int_{\mathbb{R}_{0}} \nabla l \hat{H}(t)\left(\hat{L}(t)-L^{\hat{\theta}}(t)\right) \nu(d z)\right\} d t\right] \\
& \quad=\mathbb{E}\left[\left(h(\hat{X}(T))-h\left(X^{\hat{\theta}}(T)\right)\right) \hat{\mu}(T)\right]+I_{3},
\end{aligned}
$$

where

$$
\begin{aligned}
I_{3}= & -\mathbb{E}\left[\int _ { 0 } ^ { T } \left\{\left(\hat{g}(t)-g^{\hat{\theta}}(t)+\hat{f}(t)-f^{\hat{\theta}}(t)\right) \mu(t)+\frac{\partial \hat{H}}{\partial y}(t)\left(\hat{Y}(t)-Y^{\hat{\theta}}(t)\right)\right.\right. \\
& \left.\left.+\frac{\partial \hat{H}}{\partial k}(t)\left(\hat{K}(t)-K^{\hat{\theta}}(t)\right)+\int_{\mathbb{R}_{0}} \nabla_{l} \hat{H}(t)\left(\hat{L}(t)-L^{\hat{\theta}}(t)\right) \nu(d z)\right\} d t\right] .
\end{aligned}
$$

By the concavity of $h$ and using the Itô formula again, we get

$$
\begin{aligned}
I_{2}=\mathbb{E} & {\left[\left(h(\hat{X}(T))-h\left(X^{\hat{\theta}}(T)\right)\right)(1+\hat{\mu}(T))\right]+I_{3} } \\
\geq \mathbb{E} & {\left[\left(\hat{X}(T)-X^{\hat{\theta}}(T)\right) h^{\prime}(\hat{X}(T)(1+\hat{\mu}(T))]+I_{3}\right.} \\
=\mathbb{E} & {\left[\left(\hat{X}(T)-X^{\hat{\theta}}(T)\right) \hat{\varphi}(T)\right]+I_{3} } \\
=\mathbb{E} & {\left[\int _ { 0 } ^ { T } \left\{\hat{\varphi}(t) d\left(\hat{X}(t)-X^{\hat{\theta}}(t)\right)+\left(\hat{X}(t)-X^{\hat{\theta}}(t)\right) d \hat{\varphi}(t)\right.\right.} \\
& \left.\left.+\left(\hat{\sigma}(t)-\sigma^{\hat{\theta}}(t)\right) \hat{\psi}(t)+\int_{\mathbb{R}_{0}}\left(\hat{\gamma}(t)-\gamma^{\hat{\theta}}(t)\right) \hat{\phi}(t, z) \nu(d z)\right\} d t\right]+I_{3} \\
=\mathbb{E}\left[\int _ { 0 } ^ { T } \left\{-\frac{\partial \hat{H}}{\partial x}(t)\left(\hat{X}(t)-X^{\hat{\theta}}(t)\right)+\left(\hat{b}(t)-b^{\hat{\theta}}(t)\right) \hat{\varphi}(t)\right.\right. & \\
& \left.\left.+\left(\hat{\sigma}(t)-\sigma^{\hat{\theta}}(t)\right) \hat{\psi}(t)+\int_{\mathbb{R}_{0}}\left(\hat{\gamma}(t)-\gamma^{\hat{\theta}}(t)\right) \hat{\phi}(t, z) \nu(d z)\right\} d t\right]+I_{3} .
\end{aligned}
$$

Hence

$$
\begin{aligned}
I_{1}+I_{2}=\mathbb{E}[ & \int_{0}^{T}\left\{\hat{H}(t)-H^{\hat{\theta}}(t)-\left(\frac{\partial \hat{H}}{\partial x}(t)\left(\hat{X}(t)-X^{\hat{\theta}}(t)\right)\right.\right. \\
& +\frac{\partial \hat{H}}{\partial y}(t)\left(\hat{Y}(t)-Y^{\hat{\theta}}(t)\right)+\frac{\partial \hat{H}}{\partial k}(t)\left(\hat{K}(t)-K^{\hat{\theta}}(t)\right) \\
& \left.\left.\left.+\int_{\mathbb{R}_{0}} \nabla_{l} \hat{H}(t)\left(\hat{L}(t)-L^{\hat{\theta}}(t)\right) \nu(d z)\right)\right\} d t\right] .
\end{aligned}
$$


On the other hand, since the function

$$
(x, y, k, l, \pi) \rightarrow H(t, x, y, k, l, \hat{\theta}(t), \pi, \hat{\mu}(t), \hat{\varphi}(t), \hat{\psi}(t), \hat{\phi}(t, \cdot))
$$

is concave, we have

$$
\begin{aligned}
\hat{H}(t)-H^{\hat{\theta}}(t) & \geq \frac{\partial \hat{H}}{\partial x}(t)\left(\hat{X}(t)-X^{\hat{\theta}}(t)\right)+\frac{\partial \hat{H}}{\partial y}(t)\left(\hat{Y}(t)-Y^{\hat{\theta}}(t)\right) \\
& +\frac{\partial \hat{H}}{\partial k}(t)\left(\hat{K}(t)-K^{\hat{\theta}}(t)\right)+\int_{\mathbb{R}_{0}} \nabla_{l} \hat{H}(t)\left(\hat{L}(t)-L^{\hat{\theta}}(t)\right) \nu(d z) \\
& +\frac{\partial \hat{H}}{\partial \pi}(t)(\hat{\pi}(t)-\pi(t)) .
\end{aligned}
$$

Combining (22), (23) and the condition (16), we conclude that

$$
\begin{aligned}
J_{g}(\hat{\theta}, \hat{\pi})-J_{g}(\hat{\theta}, \pi) & \geq \mathbb{E}\left[\int_{0}^{T} \frac{\partial \hat{H}}{\partial \pi}(t)(\hat{\pi}(t)-\pi(t)) d t\right] \\
& =\mathbb{E}\left[\int_{0}^{T} \mathbb{E}\left[\frac{\partial \hat{H}}{\partial \pi}(t)(\hat{\pi}(t)-\pi(t)) \mid \mathcal{G}_{t}\right] d t\right] \\
& =\mathbb{E}\left[\int_{0}^{T} \mathbb{E}\left[\frac{\partial \hat{H}}{\partial \pi}(t) \mid \mathcal{G}_{t}\right](\hat{\pi}(t)-\pi(t)) d t\right] \\
& =\mathbb{E}\left[\int_{0}^{T} \frac{\partial}{\partial \pi} \mathbb{E}\left[\hat{H}(t) \mid \mathcal{G}_{t}\right](\hat{\pi}(t)-\pi(t)) d t\right] \geq 0 .
\end{aligned}
$$

Since this holds for all $\pi \in \Pi_{\mathcal{G}}, \hat{\pi}$ is optimal.

ii) Proceeding in the same way as in (i) we can show that if (ii) holds, then

$$
J_{g}(\hat{\theta}, \hat{\pi}) \leq J_{g}(\theta, \hat{\pi}),
$$

for all $\theta \in \Theta_{\mathcal{G}}$ and $\hat{\theta}$ is optimal.

iii) If both (i) and (ii) hold then

$$
J_{g}(\hat{\theta}, \pi) \leq J_{g}(\hat{\theta}, \hat{\pi}) \leq J_{g}(\theta, \hat{\pi}),
$$

for any $(\theta, \pi) \in \Theta_{\mathcal{G}} \times \Pi_{\mathcal{G}}$. Thereby

$$
J_{g}(\hat{\theta}, \hat{\pi}) \leq \inf _{\theta \in \Theta_{\mathcal{G}}} J_{g}(\theta, \hat{\pi}) \leq \sup _{\pi \in \Pi_{\mathcal{G}}}\left(\inf _{\theta \in \Theta_{\mathcal{G}}} J_{g}(\theta, \pi)\right) .
$$

On the other hand,

$$
J_{g}(\hat{\theta}, \hat{\pi}) \geq \sup _{\pi \in \Pi_{\mathcal{G}}} J_{g}(\hat{\theta}, \pi) \geq \inf _{\theta \in \Theta_{\mathcal{G}}}\left(\sup _{\pi \in \Pi_{\mathcal{G}}} J_{g}(\theta, \pi)\right) .
$$

Now due to the inequality

$$
\inf _{\theta \in \Theta_{\mathcal{G}}}\left(\sup _{\pi \in \Pi_{\mathcal{G}}} J_{g}(\theta, \pi)\right) \geq \sup _{\pi \in \Pi_{\mathcal{G}}}\left(\inf _{\theta \in \Theta_{\mathcal{G}}} J_{g}(\theta, \pi)\right)
$$

we have

$$
J_{g}(\hat{\theta}, \hat{\pi})=\sup _{\pi \in \Pi_{\mathcal{G}}}\left(\inf _{\theta \in \Theta_{\mathcal{G}}} J_{g}(\theta, \pi)\right)=\inf _{\theta \in \Theta_{\mathcal{G}}}\left(\sup _{\pi \in \Pi_{\mathcal{G}}} J_{g}(\theta, \pi)\right) .
$$




\section{A maximum principle for nonzero-sum games with $g$-expectations}

In this section, we study a nonzero sum stochastic differential games problem with $g$-expectation. For notational simplification, we consider only two players; it is similar for $n$ players. The control system is given as before, which is

$$
\begin{aligned}
d X(t) & =b\left(t, X(t), u_{0}(t)\right) d t+\sigma\left(t, X(t), u_{0}(t)\right) d W(t) \\
& +\int_{\mathbb{R}_{0}} \gamma\left(t, X\left(t^{-}\right), u_{1}\left(t^{-}, z\right), z\right) \tilde{N}(d t, d z), t \in[0, T], \\
X(0) & =x \in \mathbb{R}^{n} .
\end{aligned}
$$

Let $u=\left(u_{0}, u_{1}\right)=(\theta, \pi)$, where $\theta=\left(\theta_{0}, \theta_{1}\right)$ and $\pi=\left(\pi_{0}, \pi_{1}\right)$ are controls for player 1 and 2 , respectively. Let $\mathcal{G}_{t}^{1} \subseteq \mathcal{F}_{t}$ and $\mathcal{G}_{t}^{2} \subseteq \mathcal{F}_{t}$ be two sub-filtrations, representing the information available to player 1 and player 2 , respectively, and let $\Theta_{\mathcal{G}^{1}}, \Pi_{\mathcal{G}^{2}}$ be the corresponding families of admissible control processes $\theta(t)$, $\pi(t) ; t \in[0, T]$. We denote by $J_{g_{i}}^{i}(\theta, \pi), i=1,2$, the performance functionals corresponding to the two players 1 and 2 :

$$
J_{g_{i}}^{i}(\theta, \pi)=\mathcal{E}_{g_{i}}\left[\int_{0}^{T} f_{i}(t, X(t), u(t)) d t+h_{i}(X(T))\right], \quad i=1,2,
$$

where $g_{i}:[0, T] \times \mathbb{R}^{n} \times \mathbb{R}^{n} \times L^{2}(\nu) \rightarrow \mathbb{R}$ are given convex functions satisfying (4). Thus $\mathcal{E}_{g_{i}}$ represents the preference of player $i, i=1,2$. The problem is to find a control $\left(\theta^{*}, \pi^{*}\right) \in \Theta_{\mathcal{G}^{1}} \times \Pi_{\mathcal{G}^{2}}$ such that

$$
\begin{cases}J_{g_{1}}^{1}\left(\theta, \pi^{*}\right) \leq J_{g_{1}}^{1}\left(\theta^{*}, \pi^{*}\right), & \text { for all } \theta \in \Theta_{\mathcal{G}^{1}} \\ J_{g_{2}}^{2}\left(\theta^{*}, \pi\right) \leq J_{g_{2}}^{2}\left(\theta^{*}, \pi^{*}\right), & \text { for all } \pi \in \Pi_{\mathcal{G}^{2}} .\end{cases}
$$

The pair of controls $\left(\theta^{*}, \pi^{*}\right)$ is called a Nash equilibrium for the game. Note that when player 1 (resp. 2) acts with the strategy $\theta^{*}$ (resp. $\pi^{*}$ ), the best that 2 (resp. 1) can do is to act with $\pi^{*}$ (resp. $\theta^{*}$ ).

We use the same method as in the previous section, but adapted to the new situation. We now consider the following forward-backward SDEs:

$$
\left\{\begin{aligned}
d X(t)= & b\left(t, X(t), u_{0}(t)\right) d t+\sigma\left(t, X(t), u_{0}(t)\right) d W(t) \\
& +\int_{\mathbb{R}_{0}} \gamma\left(t, X\left(t^{-}\right), u_{1}\left(t^{-}, z\right), z\right) \widetilde{N}(d t, d z), \\
d Y_{1}(t)= & -\left[g_{1}\left(t, K_{1}(t), L_{1}(t, \cdot)\right)+f_{1}(t, X(t), u(t))\right] d t \\
& +K_{1}(t) d W(t)+\int_{\mathbb{R}_{0}} L_{1}(t, z) \tilde{N}(d t, d z), \\
d Y_{2}(t)= & -\left[g_{2}\left(t, K_{2}(t), L_{2}(t, \cdot)\right)+f_{2}(t, X(t), u(t))\right] d t \\
& +K_{2}(t) d W(t)+\int_{\mathbb{R}_{0}} L_{2}(t, z) \widetilde{N}(d t, d z), \\
X(0)= & X_{0}, Y_{1}(T)=h_{1}(X(T)), Y_{2}(T)=h_{2}(X(T)) .
\end{aligned}\right.
$$

The performance functionals $J_{g_{i}}^{i}(\theta, \pi), i=1,2$, now take the form:

$$
\begin{aligned}
& J_{g_{i}}^{i}(\theta, \pi)=Y_{i}(0) \\
& =\mathbb{E}\left[h_{i}(X(T))+\int_{0}^{T}\left(g_{i}\left(t, K_{i}(t), L_{i}(t, \cdot)\right)+f_{i}(t, X(t), u(t))\right) d t\right], i=1,2 .
\end{aligned}
$$


We want to find a Nash equilibrium for the game, i.e. a pair $\left(\theta^{*}, \pi^{*}\right)$, such that the inequalities (27) are satisfied.

Let us introduce two Hamiltonian functions associated with this game, namely $H_{1}$ and $H_{2}$, as follows:

$$
H_{i}:[0, T] \times \mathbb{R}^{n} \times \mathbb{R}^{n} \times \mathbb{R}^{n} \times L^{2}(\nu) \times K \times K \times \mathbb{R}^{n} \times \mathbb{R}^{n} \times \mathbb{R}^{n} \times L^{2}(\nu) \rightarrow \mathbb{R}
$$

are defined by

$$
\begin{aligned}
& H_{i}\left(t, x, y_{i}, k_{i}, l_{i}, \theta, \pi, \mu_{i}, \varphi_{i}, \psi_{i}, \phi_{i}\right)=g_{i}\left(t, k_{i}, l_{i}\right)+f_{i}(t, x, \theta, \pi) \\
& +\left(g_{i}\left(t, k_{i}, l_{i}\right)+f_{i}(t, x, \theta, \pi)\right) \mu_{i}+b(t, x, \theta, \pi) \varphi_{i} \\
& +\sigma(t, x, \theta, \pi) \psi_{i}+\int_{\mathbb{R}_{0}} \gamma(t, x, \theta, \pi, z) \phi_{i}(t, z) \nu(d z), \quad i=1,2 .
\end{aligned}
$$

We assume that $H_{i}$ is differentiable with respect to the variables $x, y_{i}, k_{i}, l_{i}$, respectively. The adjoint equations in the unknown adapted processes $\mu_{i}, \varphi_{i}$, $\psi_{i}$ and $\phi_{i}, i=1,2$, is following FBSDE:

$$
\left\{\begin{aligned}
d \mu_{i}(t) & =\frac{\partial H_{i}}{\partial y}\left(t, X(t), Y_{i}(t), K_{i}(t), L_{i}(t), \theta(t), \pi(t), \mu_{i}(t), \varphi_{i}(t), \psi_{i}(t), \phi_{i}(, \cdot)\right) d t \\
& +\frac{\partial H_{i}}{\partial k_{i}}\left(t, X(t), Y_{i}(t), K_{i}(t), L_{i}(t), \theta(t), \pi(t), \mu_{i}(t), \varphi_{i}(t), \psi_{i}(t), \phi_{i}(, \cdot)\right) d W(t) \\
& +\int_{\mathbb{R}_{\mathbb{R}}} \nabla_{l_{i}} H_{i}\left(t, X(t), Y_{i}(t), K_{i}(t), L_{i}(t), \theta(t), \pi(t), \mu_{i}(t), \varphi_{i}(t), \psi_{i}(t), \phi_{i}(, \cdot)\right) \widetilde{N}(d t, d z), \\
d \varphi_{i}(t) & =-\frac{\partial H_{i}}{\partial x}\left(t, X(t), Y_{i}(t), K_{i}(t), L_{i}(t), \theta(t), \pi(t), \mu_{i}(t), \varphi_{i}(t), \psi_{i}(t), \phi_{i}(, \cdot)\right) d t \\
& +\psi_{i}(t) d W(t)+\int_{\mathbb{R}_{0}} \phi_{i}(t, z) \widetilde{N}(d t, d z), \\
\mu_{i}(0) & =0, \quad \varphi_{i}(T)=\left(1+\mu_{i}(T)\right) h_{i}^{\prime}(X(T)) .
\end{aligned}\right.
$$

The following result is a generalization of Theorem 2.1: (As in Section 2 we let $\Theta$ and $\Pi$ denote the set of possible control values of $\theta(t), t \in[0, T]$ and $\pi(t)$, $t \in[0, T]$, respectively.)

Theorem 3.1. Let $(\hat{\theta}, \hat{\pi}) \in \Theta_{\mathcal{G}^{1}} \times \Pi_{\mathcal{G}^{2}}$ with corresponding state processes $\hat{X}(t)$, $\hat{Y}_{1}(t)$ and $\hat{Y}_{2}(t)$. Suppose there exists a solution $\left(\hat{\varphi}_{i}(t), \hat{\psi}_{i}(t), \hat{\phi}_{i}(t, z)\right), i=1,2$, of the corresponding adjoint equation (31) such that

$$
\begin{aligned}
& \max _{\pi \in \Pi} \mathbb{E}\left[H_{1}\left(t, \hat{X}(t), \hat{Y}_{1}(t), \hat{K}_{1}(t), \hat{L}_{1}(t), \hat{\theta}(t), \pi, \hat{\mu}_{1}(t), \hat{\varphi}_{1}(t), \hat{\psi}_{1}(t), \hat{\phi}_{1}(, \cdot)\right) \mid \mathcal{G}_{t}^{2}\right] \\
& =\mathbb{E}\left[H_{1}\left(t, \hat{X}(t), \hat{Y}_{1}(t), \hat{K}_{1}(t), \hat{L}_{1}(t), \hat{\theta}(t), \hat{\pi}(t), \hat{\mu}_{1}(t), \hat{\varphi}_{1}(t), \hat{\psi}_{1}(t), \hat{\phi}_{1}(, \cdot)\right) \mid \mathcal{G}_{t}^{2}\right],
\end{aligned}
$$

and

$$
\begin{aligned}
& \max _{\theta \in \Theta} \mathbb{E}\left[H_{2}\left(t, \hat{X}(t), \hat{Y}_{2}(t), \hat{K}_{2}(t), \hat{L}_{2}(t), \theta, \hat{\pi}(t), \hat{\mu}_{2}(t), \hat{\varphi}_{2}(t), \hat{\psi}_{2}(t), \hat{\phi}_{2}(, \cdot)\right) \mid \mathcal{G}_{t}^{1}\right] \\
& =\mathbb{E}\left[H_{2}\left(t, \hat{X}(t), \hat{Y}_{2}(t), \hat{K}_{2}(t), \hat{L}_{2}(t), \hat{\theta}(t), \hat{\pi}(t), \hat{\mu}_{2}(t), \hat{\varphi}_{2}(t), \hat{\psi}_{2}(t), \hat{\phi}_{2}(, \cdot)\right) \mid \mathcal{G}_{t}^{1}\right] .
\end{aligned}
$$

Moreover, suppose that, for all $t \in[0, T], H_{i}\left(t, x, y_{i}, k_{i}, l_{i}, \theta, \pi, \hat{\mu}_{i}, \hat{\varphi}_{i}, \hat{\psi}_{i}, \hat{\phi}_{i}\right)$ is concave in $x, y_{i}, k_{i}, l_{i}, \theta, \pi$ and $h_{i}(x)$ is concave in $x, i=1,2$. Then $(\hat{\theta}(t), \hat{\pi}(t))$ is a Nash equilibrium for the game. 
Proof. Proceeding as in proof of Theorem 2.1 we have

$$
\begin{aligned}
& J_{g_{1}}^{1}(\hat{\theta}, \hat{\pi})-J_{g_{1}}^{1}(\hat{\theta}, \pi)=\mathbb{E}\left[h_{1}(\hat{X}(T))-h_{1}\left(X^{\hat{\theta}}(T)\right)\right. \\
& \left.+\int_{0}^{T}\left\{\hat{g}_{1}(t)-g_{1}^{\hat{\theta}}(t)+\hat{f}_{1}(t)-f_{1}^{\hat{\theta}}(t)\right\} d t\right] \\
& =\mathbb{E}\left[h_{1}(\hat{X}(T))-h_{1}\left(X^{\hat{\theta}}(T)\right)+\left(\hat{Y}_{1}(0)-Y_{1}^{\hat{\theta}}(0)\right) \hat{\mu}_{1}(0)\right] \\
& +\mathbb{E}\left[\int _ { 0 } ^ { T } \left\{\hat{H}_{1}(t)-H_{1}^{\hat{\theta}}(t)-\left(\left(\hat{g}_{1}(t)-g_{1}^{\hat{\theta}}(t)+\hat{f}_{1}(t)-f_{1}^{\hat{\theta}}(t)\right) \hat{\mu}_{1}(t)\right.\right.\right. \\
& -\left(\hat{b}(t)-b^{\hat{\theta}}(t)\right) \hat{\varphi}_{1}(t)-\left(\hat{\sigma}(t)-\sigma^{\hat{\theta}}(t)\right) \hat{\psi}_{1}(t) \\
& \left.\left.\left.-\int_{\mathbb{R}_{0}}\left(\hat{\gamma}(t)-\gamma^{\hat{\theta}}(t)\right) \hat{\phi}_{1}(t, z) \nu(d z)\right)\right\} d t\right] \\
& =\mathbb{E}\left[\int_{0}^{T}-\left(\nabla_{x} \hat{H}(t)\left(\hat{X}(t)-X^{\hat{\theta}}(t)\right)+\nabla_{y_{1}} \hat{H}(t)\left(\hat{Y}_{1}(t)-Y_{1}^{\hat{\theta}}(t)\right)\right.\right. \\
& \left.+\nabla_{k_{1}} \hat{H}(t)\left(\hat{K}_{1}(t)-K_{1}^{\hat{\theta}}(t)\right)+\int_{\mathbb{R}_{0}} \nabla_{l_{1}} \hat{H}(t)\left(\hat{L}_{1}(t)-L_{1}^{\hat{\theta}}(t)\right) \nu(d z)\right) d t \\
& +\mathbb{E}\left[\int _ { 0 } ^ { T } \left(\left(\hat{g}_{1}(t)-g_{1}^{\hat{\theta}}(t)+\hat{f}_{1}(t)-f_{1}^{\hat{\theta}}(t)\right) \hat{\mu}_{1}(t)+\left(\hat{b}(t)-b^{\hat{\theta}}(t)\right) \hat{\varphi}_{1}(t)\right.\right. \\
& \left.\left.+\left(\hat{\sigma}(t)-\sigma^{\hat{\theta}}(t)\right) \hat{\psi}_{1}(t)+\int_{\mathbb{R}_{0}}\left(\hat{\gamma}(t)-\gamma^{\hat{\theta}}(t)\right) \hat{\phi}_{1}(t, z) \nu(d z)\right) d t\right] \\
& +\mathbb{E}\left[\int _ { 0 } ^ { T } \left\{\hat{H}_{1}(t)-H_{1}^{\hat{\theta}}(t)-\left(\left(\hat{g}_{1}(t)-g_{1}^{\hat{\theta}}(t)+\hat{f}_{1}(t)-f_{1}^{\hat{\theta}}(t)\right) \hat{\mu}_{1}(t)\right.\right.\right. \\
& +\left(\hat{b}(t)-b^{\hat{\theta}}(t)\right) \hat{\varphi}_{1}(t)+\left(\hat{\sigma}(t)-\sigma^{\hat{\theta}}(t)\right) \hat{\psi}_{1}(t) \\
& \left.\left.\left.+\int_{\mathbb{R}_{0}}\left(\hat{\gamma}(t)-\gamma^{\hat{\theta}}(t)\right) \hat{\phi}_{1}(t, z) \nu(d z)\right)\right\} d t\right] \\
& =\mathbb{E}\left[\int _ { 0 } ^ { T } \left\{\hat{H}_{1}(t)-H_{1}^{\hat{\theta}}(t)-\left(\nabla x \hat{H}_{1}(t)\left(\hat{X}(t)-X^{\hat{\theta}}(t)\right)\right.\right.\right. \\
& +\nabla_{y_{1}} \hat{H}_{1}(t)\left(\hat{Y}_{1}(t)-Y_{1}^{\hat{\theta}}(t)\right)+\nabla_{k_{1}} \hat{H}_{1}(t)\left(\hat{K}_{1}(t)-K_{1}^{\hat{\theta}}(t)\right) \\
& \left.\left.\left.+\int_{\mathbb{R}_{0}} \nabla_{l_{1}} \hat{H}_{1}(t)\left(\hat{L}_{1}(t)-L_{1}^{\hat{\theta}}(t)\right) \nu(d z)\right)\right\} d t\right] \text {. }
\end{aligned}
$$

Since $H_{1}$ is concave in $x, y_{1}, k_{1}, l_{1}$ and $\pi$, we get,

$$
\begin{aligned}
\mathbb{E}[ & \left.\int_{0}^{T}\left(\hat{H}_{1}(t)-H_{1}^{\hat{\theta}}(t)\right) d t\right] \geq \mathbb{E}\left[\int _ { 0 } ^ { T } \left(\nabla x \hat{H}_{1}(t)\left(\hat{X}(t)-X^{\hat{\theta}}(t)\right)\right.\right. \\
& +\nabla y_{1} \hat{H}_{1}(t)\left(\hat{Y}_{1}(t)-Y_{1}^{\hat{\theta}}(t)\right)+\nabla_{k_{1}} \hat{H}_{1}(t)\left(\hat{K}_{1}(t)-K_{1}^{\hat{\theta}}(t)\right) \\
& \left.\left.+\int_{\mathbb{R}_{0}} \nabla_{l_{1}} \hat{H}_{1}(t)\left(\hat{L}_{1}(t)-L_{1}^{\hat{\theta}}(t)\right) \nu(d z)+\nabla_{\pi} \hat{H}_{1}(t)(\hat{\pi}(t)-\pi(t))\right) d t\right] .
\end{aligned}
$$


Combining the above we get

$$
\begin{aligned}
J_{g_{1}}^{1}(\hat{\theta}, \hat{\pi})-J_{g_{1}}^{1}(\hat{\theta}, \pi) & \geq \mathbb{E}\left[\int_{0}^{T} \nabla_{\pi} \hat{H}_{1}(t)(\hat{\pi}(t)-\pi(t)) d t\right] \\
& =\mathbb{E}\left[\int_{0}^{T} \mathbb{E}\left[\nabla_{\pi} \hat{H}_{1}(t)(\hat{\pi}(t)-\pi(t)) \mid \mathcal{G}_{t}^{2}\right] d t\right] .
\end{aligned}
$$

On the other hand, the condition (32) gives,

$$
\mathbb{E}\left[\nabla_{\pi} \hat{H}_{1}(t)(\hat{\pi}(t)-\pi(t)) \mid \mathcal{G}_{t}^{2}\right]=(\hat{\pi}(t)-\pi(t)) \nabla_{\pi} \mathbb{E}\left[\hat{H}_{1}(t) \mid \mathcal{G}_{t}^{2}\right]_{\pi=\hat{\pi}(t)} \geq 0
$$

Hence

$$
J_{g_{1}}^{1}(\hat{\theta}, \hat{\pi})-J_{g_{1}}^{1}(\hat{\theta}, \pi) \geq 0 .
$$

In the same way we show that

$$
J_{g_{2}}^{2}(\hat{\theta}, \hat{\pi})-J_{g_{2}}^{2}(\theta, \hat{\pi}) \geq 0,
$$

whence the desired result.

\section{Application to finance}

We now apply our result in the previous section to study the worst case model risk management problem. Firstly, we recall the definition of the convex risk measure and its relation to $g$-expectation.

Definition 4.1. Let $\mathbb{F}=L^{p}(\mathbb{P})$ for some $p \in[1, \infty]$. A convex risk measure is a functional $\rho: \mathbb{F} \rightarrow \mathbb{R}$ that satisfies the following properties:

(i) (convexity) $\rho(\lambda X+(1-\lambda) Y) \leq \lambda \rho(X)+(1-\lambda) \rho(Y) ; X, Y \in \mathbb{F}, \lambda \in(0,1)$,

(ii) (monotonicity) If $X \leq Y$ then $\rho(X) \geq \rho(Y), \quad X, Y \in \mathbb{F}$,

(iii) (translation invariance)

$$
\rho(X+m)=\rho(X)-m, \quad X \in \mathbb{F}, m \in \mathbb{R} .
$$

To connect to the above theory we give another representation of the convex risk measure in term of $g$-expectation:

Definition 4.2. The risk $\rho(\xi)$ of random variable $\xi \in \mathbb{L}^{2}\left(\mathcal{F}_{T}, \mathbb{P}\right)(\xi$ can be seen as a financial position of a trader in a financial market) is defined by

$$
\rho(\xi):=\mathcal{E}_{g}[-\xi]:=Y(0),
$$

where $Y(0)$ is the value at $t=0$ of the solution $\operatorname{BSDE}(5)$, but with $\xi$ replaced by $-\xi$. 
Suppose that the finance market consists of one risky finance asset, whose unit price is denoted by $S_{1}(t)$, and one risk-free asset, whose price at time $t$ is denoted by $S_{0}(t)$. We use the following stochastic differential equation to describe this financial market.

$$
\left\{\begin{aligned}
d S_{0}(t) & =r(t) S_{0}(t) d t ; \quad S_{0}(0)=1 \\
d S_{1}(t) & =S_{1}\left(t^{-}\right)\left[\alpha(t) d t+\beta(t) d W(t)+\int_{\mathbb{R}} \gamma(t, z) \tilde{N}(d t, d z)\right] \\
S_{1}(0) & >0
\end{aligned}\right.
$$

where $r(t)$ is a deterministic function; $\alpha(t), \beta(t)$ and $\gamma(t, z)$ are given $\mathcal{F}_{t^{-}}$ predictable functions satisfying the following integrability condition:

$$
\begin{aligned}
& E\left[\int _ { 0 } ^ { T } \left\{|r(s)|+|\alpha(s)|+\frac{1}{2} \beta(s)^{2}\right.\right. \\
& \left.\left.\quad+\int_{\mathbb{R}}|\log (1+\gamma(s, z))-\gamma(s, z)| \nu(d z)\right\} d s\right]<\infty
\end{aligned}
$$

where $T$ is fixed. We assume that

$$
\gamma(t, z) \geq-1 \quad \text { for a.a. } t, z \in[0, T] \times \mathbb{R}_{0},
$$

where $\mathbb{R}_{0}=\mathbb{R} \backslash\{0\}$. This model represents a natural generalization of the classical Black-Scholes market model to the case where the coefficients are not necessarily constants, but allowed to be (predictable) stochastic processes. Moreover, we have added a jump component. See e.g. [3] or [7] for discussions of such markets.

Let $\mathcal{G}_{t} \subseteq \mathcal{F}_{t}$ be a given sub-filtration and $\pi(t)$ be a portfolio, representing the fraction of the total wealth invested in the risky asset at time $t$. Then the dynamics of the corresponding wealth process $V^{(\pi)}(t)$ is

$$
\left\{\begin{aligned}
d V^{(\pi)}(t) & =V^{(\pi)}\left(t^{-}\right)[\{r(t)+(\alpha(t)-r(t)) \pi(t)\} d t \\
& \left.+\pi(t) \beta(t) d W(t)+\pi\left(t^{-}\right) \int_{\mathbb{R}} \gamma(t, z) \tilde{N}(d t, d z)\right] \\
V^{(\pi)}(0) & =x>0 .
\end{aligned}\right.
$$

A portfolio $\pi$ is called admissible if it is a measurable càdlàg stochastic process adapted to filtration $\mathcal{G}_{t}$ and satisfies

$$
\pi\left(t^{-}\right) \gamma(t, z)>-1 \quad \text { a.s. }
$$

and

$$
\begin{aligned}
\int_{0}^{T}\{ & |r(t)+(\alpha(t)-r(t)) \pi(t)|+\pi^{2}(t) \beta^{2}(t) \\
& \left.+\pi^{2}(t) \int_{\mathbb{R}} \gamma^{2}(t, z) \nu(d z)\right\} d t<\infty \quad \text { a.s. }
\end{aligned}
$$

The requirement that $\pi$ should be adapted to the filtration $\mathcal{G}_{t}$ is a mathematical way of requiring that the choice of the portfolio value $\pi(t)$ at time $t$ is 
only allowed to depend on the information $\left(\sigma\right.$-algebra) $\mathcal{G}_{t}$. The wealth process corresponding to an admissible portfolio $\pi$ is the solution of (42), namely

$$
\begin{aligned}
V^{(\pi)}(t) & =x \exp \left[\int _ { 0 } ^ { t } \left\{r(t)+(\alpha(t)-r(t)) \pi(t)-\frac{1}{2} \pi^{2}(t) \beta^{2}(t)\right.\right. \\
& \left.+\int_{\mathbb{R}}(\ln (1+\pi(s) \gamma(s, z))-\pi(z) \gamma(s, z)) \nu(d z)\right\} d s \\
& \left.+\int_{0}^{t} \pi(s) \beta(s) d W(s)+\int_{0}^{t} \int_{\mathbb{R}} \ln (1+\pi(s) \gamma(s, z)) \tilde{N}(d s, d z)\right] .
\end{aligned}
$$

Now we introduce a family $\mathcal{Q}$ of measures $Q_{\theta}$ parameterized by processes $\theta=\left(\theta_{0}(t), \theta_{1}(t, z)\right)$ such that

$$
d Q_{\theta}(\omega)=Z_{\theta}(T) d P(\omega) \quad \text { on } \mathcal{F}_{T},
$$

where

$$
\left\{\begin{array}{l}
d Z_{\theta}(t)=Z_{\theta}\left(t^{-}\right)\left[-\theta_{0}(t) d W(t)-\int_{\mathbb{R}} \theta_{1}(t, z) \tilde{N}(d t, d z)\right], \\
Z_{\theta}(0)=1 .
\end{array}\right.
$$

We assume that $\theta_{1}(t, z) \leq 1$ for a.a. t,z and

$$
\int_{0}^{T}\left\{\theta_{0}^{2}(s)+\int_{\mathbb{R}} \theta_{1}^{2}(s, z)\right\} d s<\infty \quad \text { a.s. }
$$

If $\theta=\left(\theta_{0}(t), \theta_{1}(t, z)\right)$ satisfy

$$
E\left[Z_{\theta}(T)\right]=1,
$$

then $Q_{\theta}$ is a probability measure. If, in addition,

$$
\beta(t) \theta_{0}(t)+\int_{\mathbb{R}} \gamma(t, z) \theta_{1}(t, z) \nu(d z)=\alpha(t)-r(t) ; \quad t \in[0, T],
$$

then $d Q_{\theta}(\omega)=Z_{\theta}(T) d P(\omega)$ is an equivalent local martingale measure. See e.g. [7], Ch.1. But here we do not assume (47) holds.

The processes $\theta=\left(\theta_{0}, \theta_{1}\right)$ which are adapted to the sub-filtration $\mathcal{G}_{t}$ and satisfy (45) and (46) are called admissible controls of the market. The families of admissible controls $\theta$ is denoted by $\Theta$.

The performance (risk) is now defined as follows:

$$
J_{g}(\theta, \pi):=\rho\left(Z_{\theta}(T) V^{(\pi)}(T)\right)=\mathcal{E}_{g}\left[-Z_{\theta}(T) V^{(\pi)}(T)\right] .
$$

We then introduce our problem to find $\left(\theta^{*}, \pi^{*}\right) \in \Theta \times \Pi$ such that

$$
J_{g}\left(\theta^{*}, \pi^{*}\right)=\mathcal{E}_{g}\left[-Z_{\theta^{*}}(T) V^{\left(\pi^{*}\right)}(T)\right]=\sup _{\theta \in \Theta}\left(\inf _{\pi \in \Pi} \mathcal{E}_{g}\left[-Z_{\theta}(T) V^{(\pi)}(T)\right]\right) .
$$

This is a stochastic differential game between the agent and the market. The agent wants to minimal her risk over all portfolios $\pi$ and the market wants to maximize the minimal risk of the agent over all "scenarios", represented by all probability measures $Q_{\theta} ; \theta \in \Theta$. 
Put

$$
d X(t)=\left[\begin{array}{l}
d X_{1}(t) \\
d X_{2}(t)
\end{array}\right]=\left[\begin{array}{c}
d Z_{\theta}(t) \\
d V^{(\pi)}(t)
\end{array}\right]
$$

Similarly as in the previous section the corresponding state process for $X(t)=\left(Z_{\theta}(t), V^{(\pi)}(t)\right), Y(t)=Y^{(\pi)}(t), K(t)=K^{(\pi)}(t), L(t, z)=L^{(\pi)}(t, z)$ in (11) becomes the following FBSDEs:

$$
\left\{\begin{aligned}
d X(t) & =\left[\begin{array}{c}
0 \\
V^{(\pi)}\left(t^{-}\right)\{r(t)+(\alpha(t)-r(t)) \pi\}
\end{array}\right] d t \\
& +\left[\begin{array}{c}
-Z_{\theta}(t) \theta_{0}(t) \\
V^{(\pi)}\left(t^{-}\right) \beta(t) \pi(t)
\end{array}\right] d W(t)+\left[\begin{array}{c}
-Z_{\theta}(t) \int_{\mathbb{R}} \theta_{1}(t, z) \\
V^{(\pi)}\left(t^{-}\right) \pi(t) \int_{\mathbb{R}} \gamma(t, z)
\end{array}\right] \widetilde{N}(d t, d z) \\
d Y(t) & =-g(t, K(t), L(t)) d t+K(t) d W(t)+\int_{\mathbb{R}_{0}} L(t, z) \tilde{N}(d t, d z), \\
X(0) & =\left[\begin{array}{c}
1 \\
V(0)
\end{array}\right], \quad Y(T)=-Z_{\theta}(T) V^{(\pi)}(T) .
\end{aligned}\right.
$$

By (14) the Hamiltonian becomes

$$
\begin{gathered}
H(t, x, y, k, l, \theta, \pi, \mu, \varphi, \psi, \phi) \\
=g(t, k, l)(1+\mu)+x_{2}\{r(t)+(\alpha(t)-r(t)) \pi\} \varphi_{2}-x_{1} \theta_{0}(t) \psi_{1} \\
+x_{2} \beta(t) \pi(t) \psi_{2}+\int_{\mathbb{R}_{0}}\left\{-x_{1} \theta_{1}(t, z) \phi_{1}(\cdot, z)+x_{2} \pi(t) \gamma(t, z) \phi_{2}(\cdot, z)\right\} \nu(d t, d z) .
\end{gathered}
$$

And the FBSDE of the adjoint processes is of the following form

$$
\left\{\begin{aligned}
d \mu(t)= & (1+\mu(t))\left[g_{k}(t, k, l) d W(t)+\int_{\mathbb{R}_{0}} g_{l}(t, k, l) \tilde{N}(d t, d z)\right], \\
d \varphi_{1}(t)= & \left\{\theta_{0}(t) \psi_{1}(t)+\int_{\mathbb{R}_{0}} \theta_{1}(t, z) \phi_{1}(t, z)\right\} d t+\psi_{1}(t) d W(t) \\
& +\int_{\mathbb{R}_{\mathbb{R}}} \phi_{1}(t, z) \widetilde{N}(d t, d z), \\
d \varphi_{2}(t)= & -\left\{(r(t)+(\alpha(t)-r(t)) \pi(t)) \varphi_{2}(t)+\beta(t) \pi(t) \psi_{2}(t)\right. \\
& \left.+\int_{\mathbb{R}_{0}} \pi(t) \gamma(t, z) \phi_{2}(t, z) \nu(d t, d z)\right\} d t \\
& +\psi_{2}(t) d W(t)+\int_{\mathbb{R}} \phi_{2}(t, z) \widetilde{N}(d t, d z), \\
\mu(0)= & 0, \varphi_{1}(T)=-(1+\mu(T)) V^{(\pi)}(T), \varphi_{2}(T)=-(1+\mu(T)) Z_{\theta}(T) .
\end{aligned}\right.
$$

Let $(\hat{\theta}, \hat{\pi})$ be candidate for an optimal control and let $\hat{X}(t)=\left(\hat{X}_{1}(t), \hat{X}_{2}(t)\right)$, $\hat{Y}(t)$ be the corresponding optimal processes with corresponding solution $\hat{\mu}(t)$, $\hat{\varphi}(t)=\left(\hat{\varphi}_{1}(t), \hat{\varphi}_{2}(t)\right), \hat{\psi}(t)=\left(\hat{\psi}_{1}(t), \hat{\psi}_{2}(t)\right), \hat{\phi}(t, \cdot)=\left(\hat{\phi}_{1}(t, \cdot), \hat{\phi}_{2}(t, \cdot)\right)$ of the adjoint equations.

We first minimize the Hamiltonian $E\left[H\left(t, x_{1}, x_{2}, y, k, l, \theta, \pi, \mu, \varphi, \psi, \phi\right) \mid \mathcal{G}_{t}\right]$ over all $\pi \in \Pi$. This gives the following condition for a minimum point $\hat{\pi}$ :

$$
\mathbb{E}\left[(\alpha(t)-r(t)) \hat{\varphi}_{2}(t)+\beta(t) \hat{\psi}_{2}(t)+\int_{\mathbb{R}_{0}} \gamma(t, z) \hat{\phi}_{2}(t, z) \nu(d t, d z) \mid \mathcal{G}_{t}\right]_{\pi=\hat{\pi}(t)}=0 .
$$


And then we maximize $E\left[H\left(t, x_{1}, x_{2}, y, k, l, \theta, \pi, \mu, \varphi, \psi, \phi\right) \mid \mathcal{G}_{t}\right]$ over all $\theta \in \Theta$. This gives the following condition for a maximum point $\hat{\theta}=\left(\theta_{0}, \theta_{1}\right)$ :

$$
\mathbb{E}\left[-\hat{X}_{1}(t) \hat{\psi}_{1}(t) \mid \mathcal{G}_{t}\right]_{\theta=\hat{\theta}(t)}=0,
$$

and

$$
\int_{\mathbb{R}_{0}} \mathbb{E}\left[-\hat{X}_{1}(t) \hat{\phi}_{1}(t, z) \mid \mathcal{G}_{t}\right]_{\theta=\hat{\theta}(t)} \nu(d z)=0
$$

We try a process $\hat{\varphi}_{1}(t)$ of the form

$$
\hat{\varphi}_{1}(t)=-f(t)(1+\hat{\mu}(t)) \hat{X}_{2}(t)
$$

with $f$ is a deterministic differentiable function. And differentiating this, we get

$$
\begin{aligned}
& d \hat{\varphi}_{1}(t)=-(1+\hat{\mu}(t)) \hat{X}_{2}(t)\left\{f^{\prime}(t)+f(t)[r(t)+(\alpha(t)-r(t)) \hat{\pi}(t)]\right. \\
& \left.\quad+f(t) \beta(t) \hat{\pi}(t) g_{k}(t, k, l)+f(t) \hat{\pi}(t) g_{l}(t, k, l) \int_{\mathbb{R}_{0}} \gamma(t, z) \nu(d t, d z)\right\} d t \\
& -\hat{\varphi}_{1}(t)\left\{\left(g_{k}(t, k, l)+\beta(t) \hat{\pi}(t)\right) d W(t)+\int_{\mathbb{R}_{0}}\left(g_{l}(t, k, l)+\hat{\pi}(t) \gamma(t, z)\right) \tilde{N}(d t, d z)\right\} .
\end{aligned}
$$

Comparing this with the equation of $\hat{\varphi}_{1}(t)$ in (53) by equating the $d t, d B(t)$ and $\widetilde{N}(d t, d z)$ coefficients respectively, we get

$$
\begin{aligned}
\hat{\psi}_{1}(t) & =-\hat{\varphi}_{1}(t)\left(g_{k}(t, k, l)+\beta(t) \hat{\pi}(t)\right), \\
\hat{\phi}_{1}(t, z) & =-\hat{\varphi}_{1}(t)\left(g_{l}(t, k, l)+\hat{\pi}(t) \gamma(t, z)\right) .
\end{aligned}
$$

Substituting $\hat{\psi}_{1}(t)$ and $\hat{\phi}_{1}(t, z)$ into (55) and (55) we get:

$$
\mathbb{E}\left[\hat{\pi}(t)\left(\beta(t)+\int_{\mathbb{R}_{0}} \gamma(t, z) \widetilde{N}(d t, d z)\right)+g_{k}(t, k, l)+\int_{\mathbb{R}_{0}} g_{l}(t, k, l) \tilde{N}(d t, d z) \mid \mathcal{G}_{t}\right]=0 .
$$

Now we try process $\hat{\varphi}_{2}(t)$ of the form

$$
\hat{\varphi}_{2}(t)=-f(t)(1+\hat{\mu}(t)) \hat{X}_{1}(t) .
$$

Differentiating this and then comparing the obtained equation with the equation of $\hat{\varphi}_{1}(t)$ in (53) by equating the $d t, d B(t)$ and $\widetilde{N}(d t, d z)$ coefficients respectively, we get

$$
\begin{gathered}
\hat{\theta}_{0}(t) \mathbb{E}\left[\beta(t) \mid \mathcal{G}_{t}\right]-\int_{\mathbb{R}} \hat{\theta}_{1}(t, z) \mathbb{E}\left[\gamma(t, z) \mid \mathcal{G}_{t}\right] \nu(d z) \\
=E\left[\left(\alpha(t) \mid \mathcal{G}_{t}\right]-r(t)+\mathbb{E}\left[\beta(t) g_{k}(t, k, l)+\int_{\mathbb{R}_{0}} \gamma(t, z) g_{l}(t, k, l) \nu(d z) \mid \mathcal{G}_{t}\right] .\right.
\end{gathered}
$$

We have proved: 
Theorem 4.3. The optimal portfolio $\hat{\pi} \in \Pi$ for the agent is given by

$$
\mathbb{E}\left[\hat{\pi}(t)\left(\beta(t)+\int_{\mathbb{R}_{0}} \gamma(t, z) \tilde{N}(d t, d z)\right)+g_{k}(t, k, l)+\int_{\mathbb{R}_{0}} g_{l}(t, k, l) \tilde{N}(d t, d z) \mid \mathcal{G}_{t}\right]=0,
$$

and the optimal measure $Q_{\hat{\theta}}$ for the market is to choose $\hat{\theta}=\left(\hat{\theta}_{0}, \hat{\theta}_{1}\right)$ such that

$$
\begin{gathered}
\hat{\theta}_{0}(t) \mathbb{E}\left[\beta(t) \mid \mathcal{G}_{t}\right]-\int_{\mathbb{R}} \hat{\theta}_{1}(t, z) \mathbb{E}\left[\gamma(t, z) \mid \mathcal{G}_{t}\right] \nu(d z) \\
=E\left[\left(\alpha(t) \mid \mathcal{G}_{t}\right]-r(t)+\mathbb{E}\left[\beta(t) g_{k}(t, k, l)+\int_{\mathbb{R}_{0}} \gamma(t, z) g_{l}(t, k, l) \nu(d z) \mid \mathcal{G}_{t}\right] .\right.
\end{gathered}
$$

Remark. In the case when $\mathcal{E}_{t}=\mathcal{F}_{t}$ for all $t$ and the cost function is expressed by linear expectation of risk (i.e. $g=0$ ), this was proved in [8]. In this case the interpretation of this result is the following: The market maximizes the minimal risk of the agent by choosing a "scenario" (represented by a probability law $d Q_{\theta}=Z_{\theta}(T) d P$ ) which is an equivalent martingale measure for the market (see (47)). In this case the optimal strategy for the agent is to place all the money in the risk free asset, i.e. to choose $\pi(t)=0$ for all $t$. In our case an analogue result is obtained except now the coefficients $\beta(t), \gamma(t, z)$ and $\alpha(t)$ are replaced by their conditional expectations $\mathbb{E}\left[\beta(t) \mid \mathcal{G}_{t}\right], \mathbb{E}\left[\gamma(t, z) \mid \mathcal{G}_{t}\right], \mathbb{E}\left[\alpha(t) \mid \mathcal{G}_{t}\right]$ and an extra term in the formula (62) and (62) are caused by $g$-expectation.

\section{References}

[1] T. T. K. An and B. Øksendal. A Maximum Principle for Stochastic Differential Games with Partial Information. J. Optimization Theory and Application 139 (3), 463-483, 2008.

[2] T. T. K. An, B. Øksendal and Y. Y. Okur. A Malliavin Calculus Approach to General Stochastic Differential Games with Partial Information. E-Print, Dept. of Math./CMA, University of Oslo 26,Oslo, Norway, 2008.

[3] R. Cont and P. Tankov. Financial Modelling with Jump Processes. Volum 2 av Chapman and Hall/CRC Financial Mathematics Series, London, 2004.

[4] H. Föllmer and A. Schied. Convex Measure of Risk and Trading Constraints. Finance Stochastic 2, 429-447, 2002.

[5] M. Frittelli and E. R. Gianin. Putting Order in Risk Measures. J. Banking and Finance 26, 1473-1486, 2002.

[6] S. Mataramvura and B. Øksendal. Risk Minimizing Portfolios and HJB Equations for Stochastic Differential Games. Stochastics 80, 317-337, 2008.

[7] B. Øksendal and A. Sulem. Applied Stochastic Control of Jump Diffusions. Second Edition. Springer, Berlin, 2007. 
[8] B. Øksendal and A. Sulem. A Game Theoretic Approach to Martingale Measures in Incomplete Markets. Surveys of Applied and Industrial Mathematics (TVP Publishers, Moscow) 15, 18-24, 2008.

[9] B. Øksendal and A. Sulem. Maximum Principles for Optimal Control of Forward-Backward Stochastic Differential Equations with Jumps. SIAM J. Control Optimization 48, 2945-2976, 2009.

[10] S. Peng. Backward SDE and Related g-Expectation. Backward Stochastic Differential Equations. Pitman Research Notes in Mathematics Series 364, 141-159, Longman, Harlow, 1997.

[11] S. Peng. Nonlinear Expectations, Nonlinear Evaluations and Risk Measures. Stochastic Methods in Finance, Lecture Notes in Math. 1856, 165-253. Springer, Berlin, 2004. 